\title{
A Digital Interface for the Part Designers and the Fixture Designers for a Reconfigurable Assembly System
}

\author{
Vishwa V. Kumar, ${ }^{1}$ Salik R. Yadav, ${ }^{2}$ F. W. Liou, ${ }^{1}$ and S. N. Balakrishnan ${ }^{3}$ \\ ${ }^{1}$ Department of Manufacturing Engineering, Missouri University of Science \& Technology, Rolla, MO 65409, USA \\ ${ }^{2}$ Health Care Service Corporation, Chicago, IL 60601, USA \\ ${ }^{3}$ Department of Mechanical \& Aerospace Engineering, Missouri University of Science \& Technology, Rolla, MO 65409, USA
}

Correspondence should be addressed to F. W. Liou; liou@mst.edu

Received 25 February 2013; Revised 7 June 2013; Accepted 7 June 2013

Academic Editor: Zhuming Bi

Copyright (C) 2013 Vishwa V. Kumar et al. This is an open access article distributed under the Creative Commons Attribution License, which permits unrestricted use, distribution, and reproduction in any medium, provided the original work is properly cited.

\begin{abstract}
This paper presents a web-based framework for interfacing product designers and fixture designers to fetch the benefits of early supplier involvement (ESI) to a reconfigurable assembly system (RAS). The interfacing of the two members requires four steps, namely, collaboration chain, fixture supplier selection, knowledge share, and accommodation of service facilities so as to produce multiple products on a single assembly line. The interfacing not only provokes concurrency in the activities of product and fixture designer but also enables the assembly systems to tackle the spatial and generational variety. Among the four stages of interfacing, two steps are characterized by optimization issues, one from the product customer side and the other from the fixture designer side. To impart promptness in the optimization and hence the interaction, computationally economic tools are also presented in the paper for both of the supplier selection and fixture design optimization.
\end{abstract}

\section{Introduction}

In the current scenario, immense pressure faced by manufacturing firms to meet the precariously varying demand is a fait accompli. This is so because the market always demands a very large product variety among different products manufactured by any firm. The varying trend of the product variety in a product family is sought not only across different market segments (spatial variety) but also across different generations of the same product (generational variety) [1]. Obviously, these trends of product variety are valid for almost every kind of product development process, assembly systems not being the exception. Meeting the challenges posed by two kinds of variety is even higher in the case of assembly systems as it may require huge investments in different assembly lines or imparting reconfigurability to a single assembly line.

In multistation assembly processes, fixtures play the central role as most of the assembly faults are related to them. Moreover, to impart the desired product variety and to ensure unhampered product development of a product family being assembled on a single line, the assembly fixtures are required to possess certain sophistications. These sophistications include reconfigurability [2], stability [3], and fault diagnosability [4].

Reconfigurable assembly fixtures are those on which production of multiple parts can be carried out. That is, a fixture is sought to possess reconfigurability so as to produce multiple products in a single reconfigurable assembly system [2]. Most of the previous research on assembly fixtures is concerned with fault diagnosis [5-7] owing to the fact that most of the faults occurring in assembly parts are due to failures of locating pins, clamps, or supports of the fixture. This is so because during ramp up, launch fixtures are most prone to failures [8]. However, reconfigurability is also a topic worth paying attention as it can drastically alleviate the set-up and processing costs by processing multiple parts on the same fixture [9]. This helps the firm to realize the spatial variety at lower cost and at the same time enables the firm to face severe market diversity. Further, in order to reduce the overall product development time and hence to obtain higher market share, the tool designers (such as 
die or fixture designers) are severely affected. This is so because these tool designers decide the moment start of serial production [10]. A firm using assembly lines also desires to reduce the total number of tool designers involved in the production of the product. That is, a company might be using 10 to 20 fixtures for the production of a car body, but later it may come up with a strategy to reduce it to 5 or 10 fixtures [11]. Thus, in order to meet the quest for obtaining higher market share, globalization and distribution, it is necessary to develop cooperative supply chains with lesser lead times. Keeping in mind the globalization and importance of a supply chain, a firm might prefer to buy the assembly fixtures from the outside or inside fixture suppliers; those are basically fixture designers, so that the fixture suppliers design the assembly fixtures as per demanded by the product customers. In such case, it is beneficial for the firm to reduce the number of fixture suppliers as well to a small number of system specialists. Referring to the industrial contexts, Audi has reduced its tooling suppliers by more than half over four years [12]. Thus, to meet the market diversity and varying trends, it is necessary to develop a cooperative relationship between the fixture designers and the product customers. Research addressing the collaborative relationship between fixture designing and product development is essential in the context of a reconfigurable assembly system (RAS).

This paper addresses the problem of developing collaborated fixture design and product development processes by adopting a web-based framework. The developed webbased framework presents a strategy to impart a cooperative approach to concurrently perform fixture design and assembly line production. The paper opens a way to integrate the process chains of the product development and fixture designers through the web for a RAS. The web-based framework presented hereby is termed as cyberdesigning of RASs.

Traditionally, the product development in assembly system and fixture making is resolved separately. However, for developing a product family on a single assembly line the fixture design must be in cooperation with product design; enabling a fixture to be suitable for multiple part models needs reconfigurability of the fixture $[13,14]$. The collaboration between product and fixture designers enables the assembly system to excel in the longer run because locating and clamping elements in the fixtures and their positions depend upon the work-piece design $[15,16]$. Recently, concurrent engineering has emerged to be a great tool for reviewing and coordinating different aspects in the product life cycle. Several researchers have laid emphasis on the coordination requirements in product development. However, most of their work is directed towards coordinating activities: machining processes $[17,18]$, stamping product development [10], and sheet metal forming [19]. Though few researchers have proposed that activities in product development and tool designing can be coordinated through intelligent systems [20], computer aided design [21], and so forth, the coordination of activities with reference to the context of product design and fixture design remains sparsely attended.
Recently, a few researchers have advocated the use of the internet in coordination of various activities thorough knowledge exchange, e-bidding, online communication, and so forth, thus making the internet more than an information display system. For example, product design and manufacture roles can be resolved using web technology [22-24]. Thus, it is established in the literature that web technology can be a useful tool for carrying out concurrent and intelligent decision making to provoke early supplier involvement.

Utilizing the utility of web technology, this paper presents a web-based interfacing strategy to enable an assembly system to (1) efficiently take care of the desired spatial and generational variety in the product family developed in an assembly system; (2) impart reconfigurability to the assembly system so as to produce multiple products on a single line; (3) receive uninterrupted assistance from fixture designers at various level of product development; (4) provide a tool for optimal supplier selection in stipulated time frame; (5) create a web interface for mandatory knowledge share between the assembly system and fixture designer; and (6) present a heuristic for optimizing fixture design in stipulated time frames.

The rest of the paper is organized as follows. Section 2 explains various quests, scopes, and advantages of coordinating product development in assembly system and fixture design. It is followed by the web-based interfacing framework for product customer and fixture designers in Section 3. Later, Section 4 illustrates the optimization issues in the web-based interaction. Finally, the paper is concluded in Section 5.

\section{Inspecting Collaborated Fixture Design and Product Development Processes}

\subsection{Integration of Fixture Design and Product Development.} The overall process chain for developing a product on an assembly comprises of product design, assembly process planning, fixture design, and manufacturing planning. The design of the assembly fixture determines whether it can support multiple parts or not. For any assembly line, the product development activities and fixture design are closely related, and in the case of RASs, it is even more crucial [2]. As changing characteristics of parts alters the design of fixtures, so the reconfigurability of assembly systems mainly depends upon the reconfigurability of the fixtures. Thus, the link between the product development and fixture design is in fact the link between product design and serial production. Figure 1 portrays the interrelationship between the activities of product design and fixture design.

In this paper, it is assumed that the fixture supplier realizes the design of the fixtures as per the demands of the product customer. The fixture supplier cum designer will be generically called fixture designer throughout this paper. In the reconfigurable assembly system, sequential order processing is carried out where finished and prepared documents are formerly transferred to an internal or external fixture designer after the completion of the product development. In fact, the fixture designer has to design and build a manufacturing resource without perturbing the 


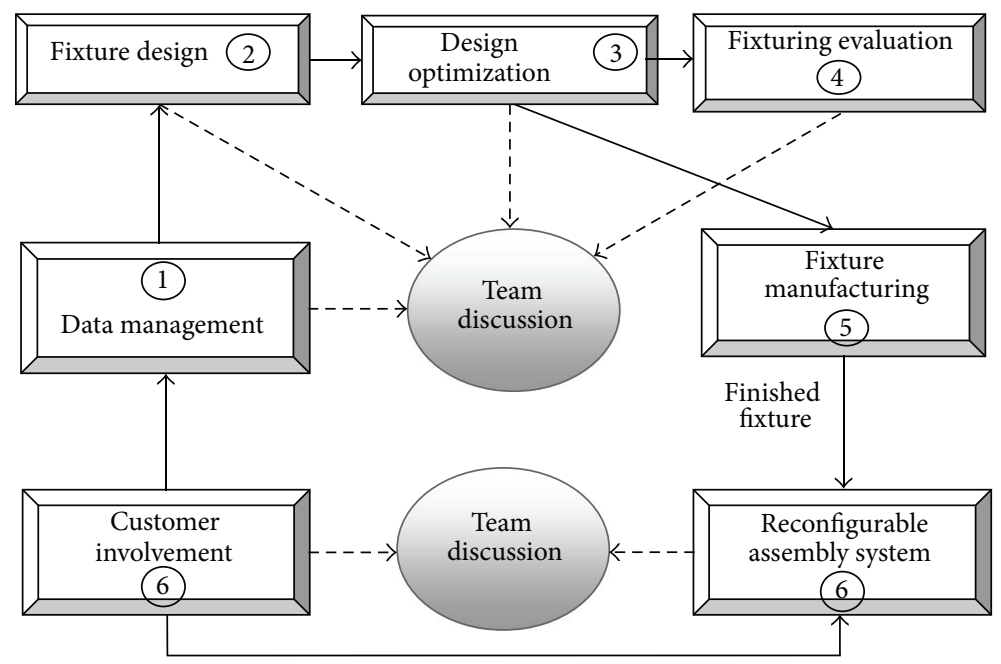

FigURE 1: Relationship between product development and fixture development.

fixture-based design of the product. This aspect portrays that a missing communication between the product and fixture development may cause various inconsistencies. These inconsistencies include poor assembling properties of the part in spite of the functional ability, expensive assembling of the part, and suitability of the fixture for multiple parts, and so forth.

The integrated fixture design and product development can reduce the development cycle time. This is possible as performing preliminary designing of the assembly part and selection of assembly fixtures concurrently instead of waiting for the complete design of the assembly part can save an enormous amount of time. Moreover, improvement in development quality also becomes viable due to consideration of potential quality problems at earlier design stages. Though integrated fixturing and product development are promising, it requires very close cooperation between part assembler and fixture designer. Figure 2 portrays integration schema for the product and fixture design.

\subsection{Synergizing Relationship between Product Customer and} Fixture Designer. Considering the importance of communication between reconfigurable assembly systems and the external or internal fixture designers, the information regarding the parts to be assembled on the fixture, such as number of parts and tooling configuration of each part, are crucial for both the product developers and the fixture designers. Once this information is received by the fixture designers, they utilize it to design the assembly fixtures. However, they also tend to optimize the design of the assembly fixtures, as discussed in the next section, which further increases the criticality of the cooperation. Moreover, the assembly process carried out in an RAS is a complex process requiring large interaction between assembly process variables such as deciding assembly robots, welding guns, and grouping of tasks. This is so because in RASs, two similar parts (say car windows for two different car models) may require an assembly robot while the other may require a welding gun for a particular process. This and other implications of RASs imply that developing products on reconfigurable assembly lines requires extensive knowledge of various processes, their capabilities, and limitations. That is, it is difficult to impart reconfigurability by a product designer without comprising with the product development processes, because of extensive requirement of knowledge and experience required, which is very unlikely in practical situations. At the same time, the fixture designers or fixture experts (designer cum suppliers) often use heuristics based on extensive experience and knowledge and can ultimately assist to carry out product development of a product family on an assembly line in a relatively short time. Hence, the cooperation between the fixture designers and the product development processes for an RAS can lead to critical savings in the product development time.

Making the fixture designers an arranging partner in the entire process chain of product development can result in efficient integration of product development and fixture development, if partnered with an early and active involvement. This scope can minimize the cost, time, and design faults leading to advantageous situations for both the customer and the fixture designers. Several researchers support this aspect; particularly Tang [11] and Eversheim [12] have proposed the active involvement of die makers in the case that stamping product development can lead to much improved results, thus, making the active cooperation between fixture designers and product development in RASs even more promising. Early integration of the customer process may lead to an earlier start of the fixture designing activities as well as a shift between the activities in the activities processed. However, an early integration is not an easy job in the context of global manufacturing, that is, when the fixture designers are geographically dispersed at large distances. In such cases, the cooperation between the fixture designers (designers cum suppliers) with the product designers and hence the concurrent performance of preliminary product design processes and fixture designer selection can be accomplished using a web-based environment. This paper presents a web-based framework termed "cyberdesigning of RASs" for cooperating 


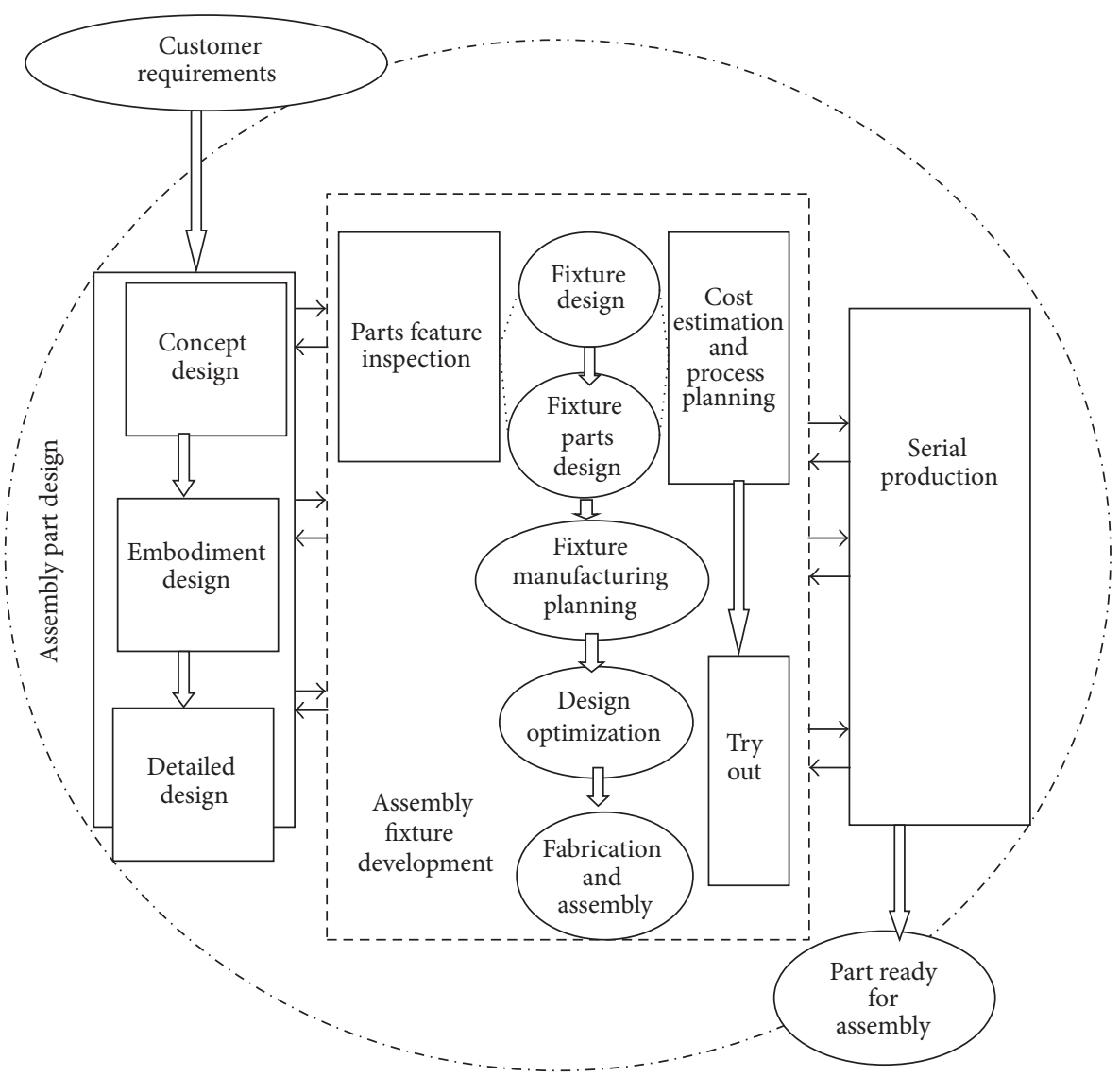

FIGURE 2: Integrated assembly fixture and part development.

and integrating the activities of fixture design and product development in an RAS as discussed in the next section.

\section{Cyberdesigning of RASs}

The web-based framework "cyberdesigning of RASs" is developed to conceptualize a cooperative integration of fixture designers with the product development of a product family on a single assembly line. In this framework, the activities in fixture design or the activities of fixture designers are juxtaposed with product development activities regarding the product family on the assembly line. This juxtaposition is accomplished by interfacing the product customer and fixture designer through the framework "cyberdesigning of RASs" as shown in Figure 3.

This framework performs the following functions for interfacing the product customer's and fixture designer's activities.

(a) Collaboration chain: The collaboration between product customer and fixture designer can be done using a bill-of-materials-based partnership chain.

(b) Fixture designer selection: A fixture designer (designer cum supplier) selection module can be developed to select appropriate fixture designers. (c) Information sharing: Information sharing includes facilities for know-how, data, and experience exchange between the RAS and the fixture designers.

(d) Service facilities: The cyberenvironment for fixture design and product assembly can be well supported by providing different functions such as user login management, online information exchange, and website administration.

All of these functions together constitute the job accomplished by the web-based interface "cyberdesigning of RASs." The descriptions of the different tasks of this interface are discussed in the following subsections.

3.1. Collaboration Chain. The collaboration chain can be defined as a partial supply chain model, consisting of basic interface between the fixture designer and the product customer. This two-stage supply chain can be represented using the notion of the generic bill of material (GBOM) of the involved product family. Though the market desires a very diverse product family, huge savings in manufacturing costs can be obtained via commonalizing some parts and differentiating others. For example, in a car body assembly system, the manufacturer can choose to differentiate the part "car bonnet" for different market segments, whereas it can offer the same part "car window" to each of the market 


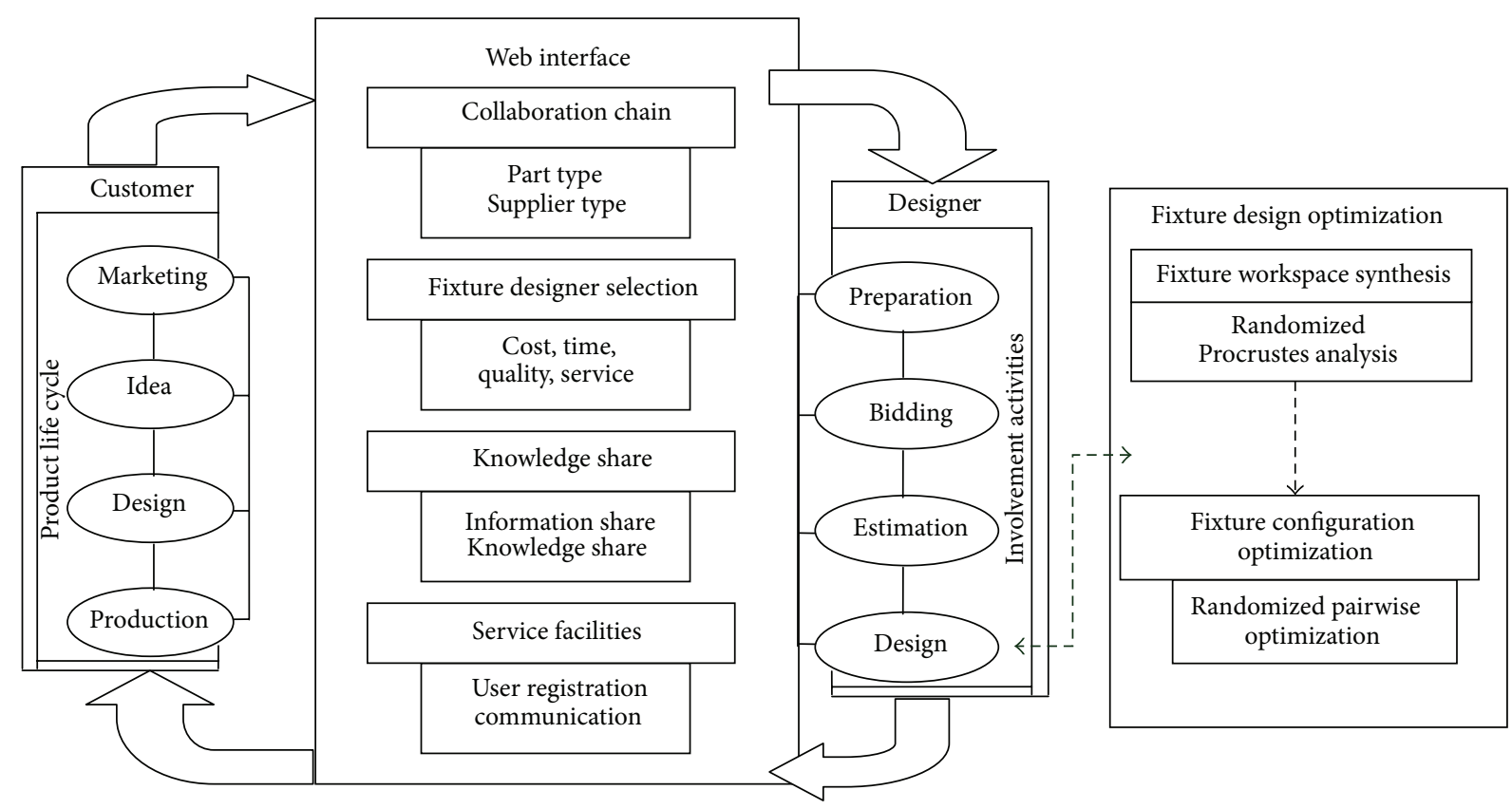

FIGURE 3: Prototype of web-based interface for product customer and fixture designer.

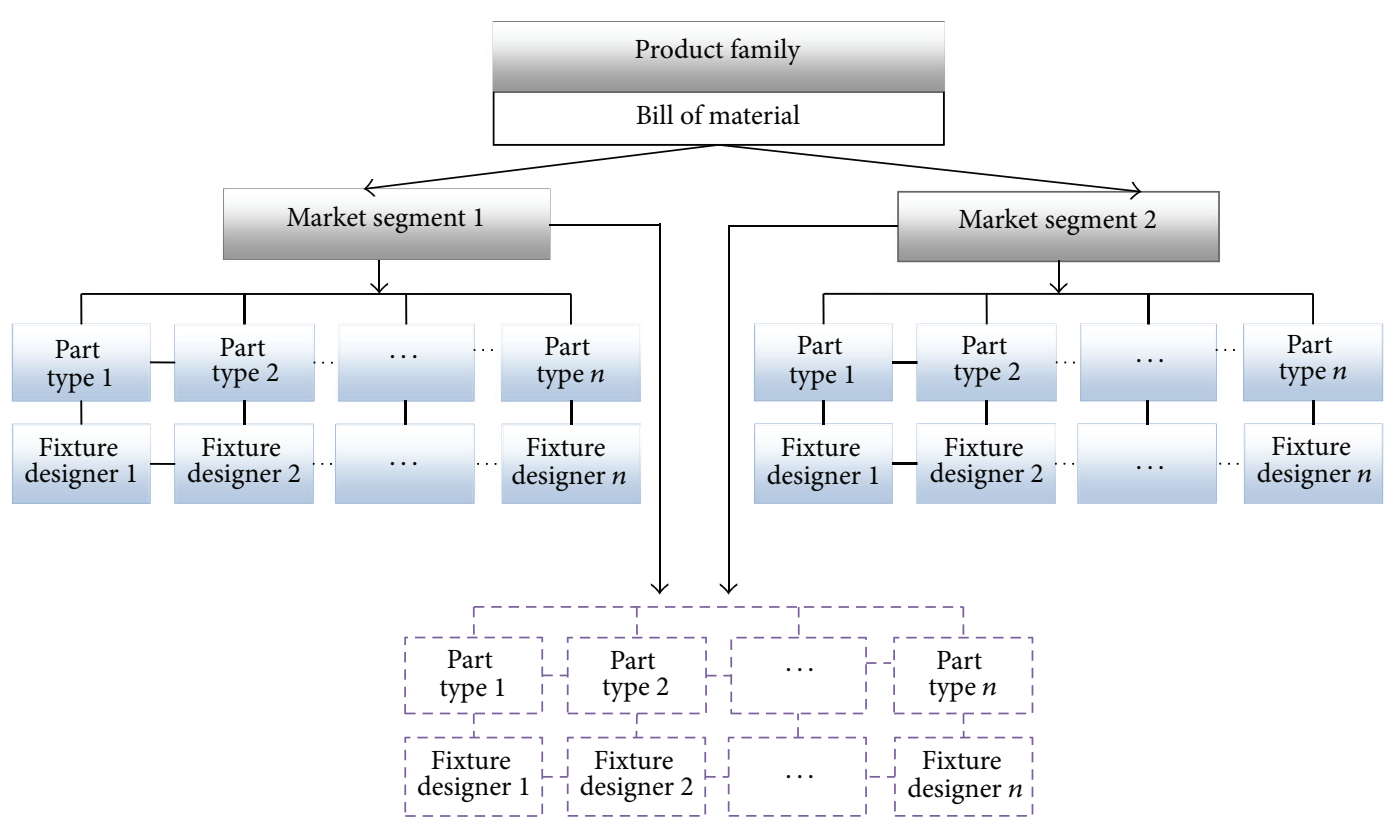

FIGURE 4: A GBOM-based collaboration chain: dotted boxes represent common parts, and solid ones indicate differentiated parts across two-market segments.

segments. Consequently, the need for the reconfigurable assembly fixture is due with the differentiated part types only.

Figure 4 portrays a GBOM employed for the collaboration chain process. In the collaboration chain process, first the customer requirements are obtained and the members of product family are planned. Then, reconfigurable and nonreconfigurable fixtures are decided as per the common or different part models to be assembled on a fixture. This is followed by the selection of individual fixture designers. For this purpose, web-based frameworks such as WeBid exist in the literature which uses BOM-based product models to ensure early supplier involvement $[25,26]$. Though BOMbased collaboration chains can bring about early supplier involvement in general product development, in case of reconfigurable assembly systems it is very complex. This is because of the very high level of responsibility and execution for technical and organizational coordination with the customers. In such case, selection of adequate fixture and tool suppliers requires advertisement for each tool or fixture. Moreover, the amendments in initially planned schedule may 


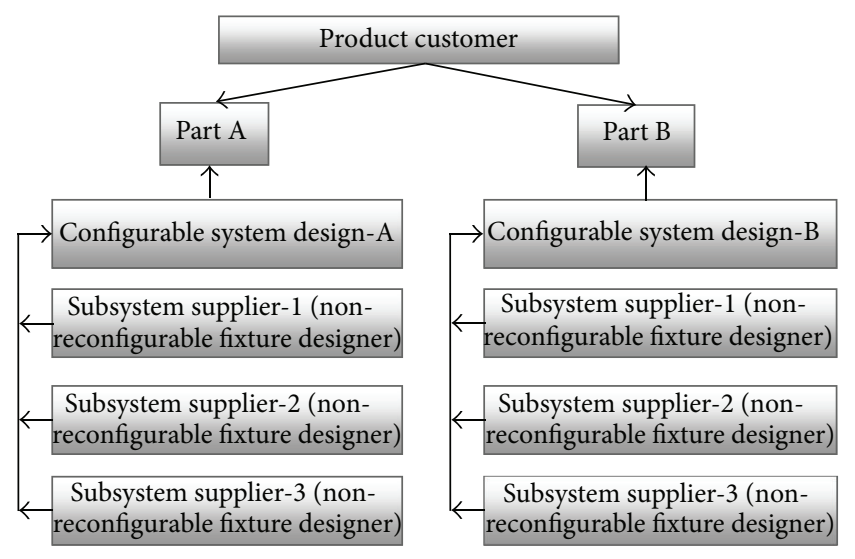

FIGURE 5: Conceptualization of virtual supplier for building multilevel collaboration chain.

raise the costs in case of multiple fixtures considered at a time, thus making the job of customer cumbersome as the number of fixture increases.

Moreover, the numbers of fixtures or tool suppliers are desired to be reduced, particularly in the automobile industry [10], so as to alleviate the costs incurred in the procurement of fixtures and other tools. An endeavour in this direction inspired from Eversheim [12] can be brought about by decreasing the number of suppliers in direct communication with the product manufacturer. This can be done by involving intermediate fixture and tool suppliers.

This paper utilizes the notions of system supplier, reconfigurable fixture designer cum supplier, and nonreconfigurable fixture designers cum supplier together forming a virtual supplier as shown in Figure 5. A system supplier is a reconfigurable assembly fixture designer meant for differentiated part types across the product family, whereas subsystem suppliers are the nonreconfigurable fixture designers meant for common part types across the product family. The subsystem suppliers together with the system suppliers constitute a virtual supplier by simultaneously contributing in the complete fixture development. It results in two improvements in the product manufacturer-fixture designer relationship. These improvements include reduction in the number of directly interacting fixture designers and transfer of overall job of fixture designing from the product manufacturer to the system supplier.

3.2. Fixture Designer Selection. For early supplier involvement, the selection of the right fixture designer is a critical issue. The criticality in the fixture designer selection lies in that a set of conflicting criteria are to be considered to realise the effective selection. These criteria range mainly in the form of technical requests along the process chain of development, fixture design and tool production, assembly, optimization, and so on. The fixture designer selection problem addressed in this paper is a multiple supplier selection problem to obtain an optimal set of fixture designers in the multilevel collaboration chain. The fixtures to be used in RAS are classified, as mentioned earlier, into reconfigurable and nonreconfigurable fixtures required, respectively, for differentiated and common part types such as components of car bodies. The two kinds of fixtures are supplied, respectively, by system and subsystem supplier. The complexity of this problem may vary from problem instance to problem instance as there may be several alternative fixture designers for the same fixture or one fixture designer may develop more than one fixture. Nonetheless, the fixture maker selection problem in this paper is concerned with the selection of the sequence of the suppliers.

The formulation of the fixture designer selection problem is inspired form Gupta and Nagi [27], Sha and Che [28], and Lin and Wang [29]. The criteria considered in the supplier selection problem can be cost, lead time, service level, quality, and so forth. The contribution of these criteria with respect to the optimality in the supplier sequence depends upon the weight or priority assigned to these criteria. They proposed heuristics based on explicit enumeration which can be used to find the optimal combination of system and subsystem assembly fixture suppliers.

Let $S_{j k}^{l}$ be the set of parts for which the $l$ th fixture designer develops the fixture at the plant $j^{l}$ for fixture type $k^{l}$. Fixture type means reconfigurable, nonreconfigurable, large size, medium size, or small size fixtures. Then, the fixture maker selection problem can be formulated as

$$
\text { Maximize } \begin{gathered}
\sum_{l=1}^{n}\left[w_{1} \cdot C_{j k}^{i}\left(S_{j k}^{l}\right)+w_{2} \cdot \Gamma_{j k}^{i}\left(S_{j k}^{l}\right)\right. \\
\left.+w_{3} \cdot Q_{j k}^{i}\left(S_{j k}^{l}\right)+\cdots\right]
\end{gathered}
$$

Subject to

$$
\begin{aligned}
& \left(s_{j k}^{l}\right) \cap\left(S_{j k}^{m}\right)=\varphi, \\
& \operatorname{Card}\left(\bigcup_{l=1}^{n(V)}\left(S_{j k}^{l}\right)\right),
\end{aligned}
$$

where $w_{1}, w_{2}$, and $w_{3}$ are the priorities or weights of the different criteria considered in the objective function. Solving the above formulated problem means finding a feasible combination of fixture designer defined by

$$
\left\langle\left(j^{l}, k^{l}, S_{j k}^{l}\right)\right\rangle_{l=1}^{n(V)}
$$

with " $n$ " being the total number of the of fixture designers in the $V$ th combination. $C_{j k}^{i}\left(S_{j k}^{l}\right), \Gamma_{j k}^{i}\left(S_{j k}^{l}\right)$ and $Q_{j k}^{i}\left(S_{j k}^{l}\right)$ are the ranks of the $l$ th fixture designer with respect to cost, lead time, and quality, respectively. For enumerative purposes, each of the criteria, whether qualitative or quantitative, needs to be quantified. For this purpose, a normalized rank or rating is used to convert the quantitative or qualitative criteria as follows:

$$
R= \begin{cases}\frac{(V-L L)}{(\mathrm{UL}-\mathrm{LL})} & \text { criteria are to be maximised } \\ \frac{(\mathrm{UL}-V)}{(\mathrm{UL}-\mathrm{LL})} & \text { criteria are to be minimised, }\end{cases}
$$


TABLE 1: Brief sketch of knowledge share components between part designer and fixture designer.

\begin{tabular}{|c|c|c|c|c|c|}
\hline \multirow[b]{2}{*}{ Part design activities } & \multicolumn{5}{|c|}{ Fixture design activities } \\
\hline & $\begin{array}{c}\text { Material cost } \\
\text { estimation }\end{array}$ & $\begin{array}{l}\text { Fixture cost } \\
\text { estimation }\end{array}$ & $\begin{array}{l}\text { Process } \\
\text { planning }\end{array}$ & $\begin{array}{c}\text { Fixture design } \\
\text { optimization }\end{array}$ & $\begin{array}{c}\text { Fixture model } \\
\text { design }\end{array}$ \\
\hline \multicolumn{6}{|l|}{ Requirements of each market segment } \\
\hline \multicolumn{6}{|l|}{ Commonality/differentiation decision } \\
\hline Material selection & $\mathrm{X}$ & $\mathrm{X}$ & & & \\
\hline Concept generation & $\mathrm{X}$ & & & & \\
\hline Individual parts configuration & & $\mathrm{X}$ & & $\mathrm{X}$ & $\mathrm{X}$ \\
\hline Tooling elements and clamps characterization & $\mathrm{X}$ & $\mathrm{X}$ & & $\mathrm{X}$ & $\mathrm{X}$ \\
\hline Dimension specification & & $\mathrm{X}$ & $\mathrm{X}$ & & $\mathrm{X}$ \\
\hline Generation of part/parts for assembly & & & & & $\mathrm{X}$ \\
\hline
\end{tabular}

where $R$ is the normalized rank or rating and $V$ is the estimated value of the quantitative or qualitative criteria converted to a quantitative value. UL is the upper limit and $\mathrm{LL}$ is the lower limit.

\subsection{Knowledge Sharing}

3.3.1. Need for Knowledge Sharing. For effective realization of early supplier involvement in the process chain, the knowledge share between the product designer and the fixture maker is the key factor. The term knowledge sharing includes both the design knowledge as well as design information sharing. The product development cycle comprises of different steps such as conceptual design steps, embodied design steps, and detailed design stage. Table 1 reveals knowledge sharing components between the part designer and fixture designer. The emphasis utilizing ESI should be to have fixture designer involvement at as many steps as possible. During the conceptual design steps, the lack of exhaustive product information may trigger the fixture designer to hesitate to specify all the workholding devices and tools that are required, thus making it difficult to perform exact fixture cost calculation. The fixture designers can help at different stages of product development cycle in the following way.

In the proposed web-based framework,

(a) the customers' verbal instructions are utilized in making a conceptual sketch of or model to generate concept parts (differentiated or common) and material selection. The involvement of fixture designers at this stage can help in identifying the most suitable material as per the sheet metal properties, forming qualities, and cost requirements.

(b) At the embodiment design stage, that is, during the part configuration processes, the assessment of fixture's cost, part's fastening properties, and utilization of previously made sketches can be well aided by the fixture designers. The cost evaluation of the previously designed part sketch is the most crucial part which is done on the configuration level.

(c) During the detailed design stage, part shape refinement, dimension specification, tolerance specifications, and clamping or tooling location specification is done. The part geometry is the most important aspect to be attended to from the side of the part designer. This is because, in assembly systems, the geometry features determine not only assembling properties but also the functional requirements of the part. For example, holes in the parts are used for different purposes such as fastening, guiding and aligning, or reducing the weight of the part. For fixture designers, part design determines the configuration of the assembly fixture designed by them. In the case of system suppliers, they have to make reconfigurable fixtures for which they require tooling or clamping specifications. They tend to minimize the fixture workspace envelope (FWE) for which they may use several alternative heuristics. The minimization of FWE during the fixture designing not only saves costs incurred during physical development of the fixture but also takes lesser space in the assembly systems. Moreover, it is also sought that the part design should be functionally acceptable as well as compatible with selected assembly processes so as to yield lower cost, shorter lead times, and higher quality.

The achievement of aforementioned objectives requires the consideration of the fixture type, number of parts assembled on a fixture, number of fixtures, and fixture development cost with respect to the part feature traits such as feature form complexity, number of tool locating elements, size, tolerance, and so forth. This requires a sound knowledge sharing between the product designer and the fixture designer. The proposed framework strives to resolve this issue by adopting any of the two knowledge sharing techniques as discussed below.

3.3.2. Mode of Knowledge Sharing. After selection of the right fixture designer for the assembly system, the early supplier involvement gets initiated from the organizational point of view. What remains yet to be materialized is to create a cooperative environment between the product designer and fixture designer. The next step in this direction, in the cyberdesigning of RASs, which follows the fixture supplier selection, is the knowledge share the quest for which is mentioned in the previous subsection. The knowledge share 


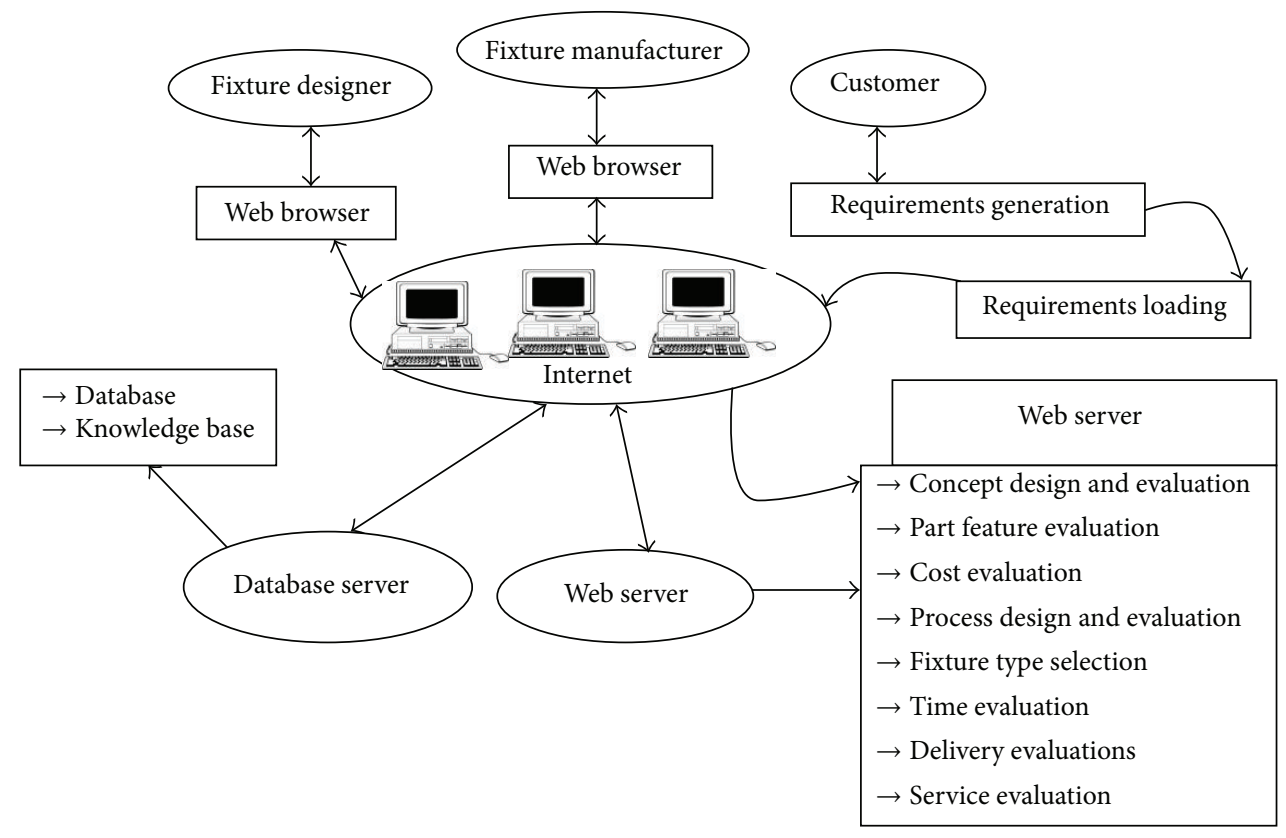

FIGURE 6: The knowledge share through web-based services in reconfigurable assembly line.

between the fixture designer and the product designer can be accomplished by either using knowledge bases furnished by fixture designers or by availing an online communication environment for messaging and data exchange between product designers and fixture designers. The former mode of knowledge share is called asynchronous knowledge share, while the latter one is known as synchronous knowledge share.

In the asynchronous knowledge share, the fixture designer provides its knowledge in web-based knowledge bases. The knowledge base can contain knowledge such as rules, case studies, heuristics, and demonstrations. Moreover, web-based design services can also be availed through the knowledge for concept design and evaluation, part design evaluation, assembly process design and evaluation, fixture set selection, and so forth, for analyzing different stages in developing the product family at the reconfigurable assembly line. Several design problems can be handled using the web-based services equipped with the knowledge base of the fixture designers. During part geometry design, the product designer can receive various options and consequences for cost, processes, and fixture design for reconfigurable as well as nonreconfigurable fixtures. These design services can be utilized to integrate the fixture designer with product development on a reconfigurable assembly system. High level information about knowledge object known as the metadata can be used to perform knowledge mining, searching and navigation. The search and navigation can be performed by adding keywords to the metadata so that specific searches can also be done. The user can browse through the keywords and explore into the knowledge base. For this purpose, Java Applet can be utilized to provide an interface for realizing the knowledge share between the product designer and the fixture designer which is clearly a future scope of research.
The mechanism of asynchronous knowledge share is shown in Figure 6.

In synchronous knowledge share, an agent communication platform based on Java Agent Template Lite [30] can be built to enable online communication between the product designer and the fixture designer. A basic infrastructure is fetched by JATLite where an agent registration is done with agent registration route (AMR) by the way of name and passwords. The agent also transfers files and invokes other programs or actions on the computer. Moreover, it can also accomplish the tasks of wrapping existing part and fixture design programmes by enabling an automatic communication with other programs via a front end. This research recommends that while utilizing synchronous knowledge share between the product designers for RASs and the corresponding fixture designers, the construction of agents can be facilitated by JATLite. The agent can send and receive knowledge query and manipulation language (KQML) and extensible markup language (XML) languages by sending a KQML message with XML contents to the receiving agent for its main applications. The receiving agent performs required operations according to the content of the message. It does so by interpreting the XML-based message content followed by its use of the related data for system application so as to minimize the data communication errors.

The part designers, via an agent, can use the features of the differentiated parts or the common parts across the product family as design units to be used by the fixture designers and configuration and evaluation units in the fixture design. The part features such as number of tooling elements or tool locators and parts' clamping configurations can be defined through appropriate feature templates. Meanwhile, XML document can be used to describe the part features. The fixture designer through an agent can read 
and analyse the part feature information via KQML/XML parser. After analyzing the part features, the fixture designer evaluates the part model for evaluation at design stages. The evaluations include reconfigurable evaluation, cost and space evaluation of the part design and hence the fixture. If the part model contains any design flaw, then the part designer is informed through the part design agent. Otherwise, the fixture designer starts developing the assembly fixture. The fixture designer will try to optimize the FWE of the fixture in case of reconfigurable as well as nonreconfigurable fixtures so as to minimize the fixture design and development costs incurred on his side. The optimization of fixture design not only fetches benefit to the fixture designer but also to the product development processes in the RAS. This is so because better space utilization of space in the assembly system and economic fixture will be availed for the RAS. Few heuristics exist in the literature, for optimizing fixture design. This paper also incorporates optimization aspects for fixture design as per the parts' design. Extended heuristics are presented in this paper that can be used by the fixture designer once the part design information is obtained, evaluated, and approved by the fixture designers. The next subsection discusses the optimization aspect of fixture development.

Thus, the cyberdesigning of RASs contains two optimization aspects, one prior to the knowledge share that is the fixture design selection and the other posterior to the knowledge share that is the fixture design optimization as per the requirements of parts' design. These optimization aspects are discussed with adopted heuristics and examples in the next section.

\section{Optimization Issues in Cyberdesigning of RASs}

As discussed in the previous section, the function modules of "cyberdesigning of RASs" include collaboration chain, fixture designer selection, knowledge share, service facilities, and supplementing tools. The functions of service facilities include aiding users' registrations who are nobody but the product customer and fixture designer. The service facilities are planned so that the customer/user can view the information of the supplier but cannot manipulate it; likewise is true for the fixture designer. The supplementing tools such as JATLite agents and KQML and XML languages are used to accomplish the function of online interaction between product customer and fixture designer through discussion, messaging, information upload and download, and so forth.

Among the above recited functions of "cyberdesigning of RASs," the first function module, namely, collaboration chain characterization, does not ask severely for optimization. Rather, it involves the provision of developing a skeleton of collaboration chain by the customers during the earlier interaction between the customer and the designer. GBOM representation of the product family involved can be used to present a graphical navigation of the collaboration skeleton so that it provides a crucial deciding point not only to the customer but also to the fixture designers. Via the help of the products GBOM, fixturing bids are invited by the customer and are evaluated by the fixture designer. The evaluation by the fixture designer involves capturing of the customer requirements and identification of his own role in the collaboration. The customer requirements include the description of parts such as number of tool locators, clamps, and supporters for the part. The latter part of evaluation refers to realization of the fact that whether the fixture designer can play the role of a system or subsystem supplier depending on differentiated or common part, and hence reconfigurable or nonreconfigurable fixtures are to be developed. After the characterization of collaboration chain by the customer, different fixture designers submit their bids. The evaluation of bids of the entire potential fixture design by the customer is next realized.

Thereafter, supplier selection from the potential fixture designers is to be done. This requires the optimization of different conflicting criteria for which sophisticated techniques and heuristics are needed, particularly for large size problems. This optimization issue is discussed later.

4.1. Prior-to-Knowledge Share Optimization. As discussed in the previous section, several criteria compete for consideration while accomplishing the supplier selection in an optimal way (1). Previously, the multiple-supplier selection problem, similar to our context, has been solved using explicit enumeration techniques. However, in cases involving a very large number of suppliers to be selected, it may be a cumbersome job to find the optimal solution in computationally feasible time. This paper proposes a random search metaheuristics, namely, artificial immune system (AIS) which is discussed below.

4.1.1. Artificial Immune System. Despite AIS's recent emergence, it has well established itself as a potential optimization algorithm backed by superior results it has yielded [31]. AIS incorporates clonal selection and evolutionary principles altogether so as to impart learning among individuals, or in computational terms, the random solutions [32].

It is a population-based strategy which starts with the maintenance of a population $P$ of antibodies. Each antibody is a random solution string encoding the sequence of feasible suppliers as given by (3). Obviously, the representation schema is a permutation schema encoding the index of the selected supplier and arranged in the sequence.

This population of antibodies undergoes cloning wherein each individual is cloned or copied based on its antigenic affinity (or objective function given by (1)). That is, more clones are generated for antibodies with higher affinity and vice versa. The antigenic affinity refers precisely to the string's fitness in terms of objective function or constraint satisfaction.

Thereafter, all the clones have undergone a maturation process through hypermutation whereby the stochastic rate of hypermutation is made dependent on the fitness. Precisely, rate of hypermutation is kept higher for clones with lower fitness and vice versa. The difference between hypermutation (AIS) and mutation (GA) lies in the fitness dependency of the mutation rate in case of AIS. From the hypermutated clones 
population, $P$, best individuals are selected stochastically and the whole procedure of cloning and hypermutation is iterated. AIS is shown to accomplish a search with a fair trade off between exploration and exploitation [31].

4.1.2. Implementation over a Case Study. An implementation of artificial immune system over a problem taken from Tsai [33] and converted to our context (the suppliers being fixture designer cum supplier) is presented in this section. The criteria considered in the problem are cost, lead time, delivery ability, service, and flexibility so that the objective function is given by

$$
\begin{aligned}
\operatorname{Maximize} a_{v}=\sum_{l=1}^{n} & {\left[w_{1} \cdot C_{j k}^{i}\left(S_{j k}^{l}\right)\right.} \\
& +w_{2} \cdot \Gamma_{j k}^{i}\left(S_{j k}^{l}\right)+w_{3} \cdot Q_{j k}^{i}\left(S_{j k}^{l}\right) \\
& +w_{4} \cdot D_{j k}^{i}\left(S_{j k}^{l}\right)+w_{5} \cdot \rho_{j k}^{i}\left(S_{j k}^{l}\right) \\
& \left.+w_{3} \cdot F_{j k}^{i}\left(S_{j k}^{l}\right)\right],
\end{aligned}
$$

where quantity represented by (5) is the assessment index of the $v$ th sequence of the fixture designers cum suppliers. $C_{j k}^{i}$ is the cost rating, $\Gamma_{j k}^{i}$ is the lead time rating, $Q_{j k}^{i}$ is the quality rating, $\rho_{j k}^{i}$ is the service rating, and $F_{j k}^{i}$ represents the flexibility rating of the $l$ th supplier at plant " $j$ " and fixture type " $k$ ".

Table 2 shows mandatory fixture designs required for parts of a car body panel. The search space for this problem can be represented by a search tree. The weights considered hereby are taken to be $0.449,0.133,0.129,0.028,0.203$, and 0.059 , respectively, for cost, lead time, quality, delivery, service, and flexibility. The solution space for the problem can be represented via a search tree, and the optimal solution for this problem varies from combination to combination of the weight of the six criteria. For the abovementioned AIS algorithm is coded over this problem on $\mathrm{C}++$ compiler and run till the optimal result is obtained. The optimal sequence found is $(1,1,\{\operatorname{LEP} 01, \operatorname{LEP} 02\})_{1-}(6,2,\{\operatorname{MIP} 04\})_{2-}(2,3$, $\left\{\right.$ SIP 03, SIP 05\}) . $_{\text {. }}$

The solution for this problem is found after 100 iterations and is more promising on large data sets as compared to explicit enumeration in terms of computational time.

4.2. Posterior-to-Knowledge Share Optimization. Once the fixture designer is selected and part design, fixture designer capabilities, and so forth get realized through knowledge share, the selected fixture designers optimize the design of the fixture as per the parts associated with the fixture. Information like part configuration (tooling elements, tool locators) is utilized for this purpose. However, in order to reduce fixture development cost, fixture workspace envelope, and so forth, the fixture designer tends to optimize the fixture design. With reference to speedy and compact interaction to enable web-based cooperation, the viability of the heuristics to yield optimal fixture design used by the fixture designer in a stipulated time frame is a crucial issue. This paper extends
TABLE 2: Fixture designer alternatives for a car body panel.

\begin{tabular}{lcc}
\hline Part & Fixture configuration type & Fixture designer options \\
\hline LEP 01 & 1 (reconfigurable fixture) & 1,3 \\
LEP 01 & 1 (reconfigurable fixture) & 1,6 \\
SIP 03 & 3 (nonreconfigurable fixture) & $2,4,6$ \\
MIP 04 & 2 (nonreconfigurable fixture) & $5,6,7$ \\
SIP 05 & 3 (nonreconfigurable fixture) & $2,8,9$ \\
\hline
\end{tabular}

a deterministic heuristic to optimize fixture configuration that can be used by the fixture designer via agent or other software to yield quality solutions in real time. The following subsection presents the extended stochastic metaheuristics for obtaining a near optimal/optimal fixture design.

4.2.1. Stochastic Procrustes-Based Pairwise Optimization. Lee et al. [34] has presented a genetic algorithm-based workspace synthesis analysis for fixturing of a stamping part family. They proposed to minimize the space required by each locator to produce a part family and thereby synthesizing the single fixture workspace. Though their approach substantially reduces the fixture workspace an analytical elimination of unlikely sets of solutions is missing that may impart swiftness to the workspace minimization. Moreover, the number of variables involved in minimization is directly proportional to the number of locators and parts causing the algorithm to consume very large amounts of time.

Kong and Ceglarek [2] proposed a methodology containing Procrustes and pairwise optimization. They utilized Procrustes analysis for workspace synthesis by obtaining best superposition of different configurations corresponding to different parts. The Procrustes analysis uses a least squares method with isotropic scaling, rigid translation, and rotation. It accomplishes preliminary fixture configuration design by best matching one configuration (set of points with multidimensions) to the others. For multiple parts, generalized Procrustes analysis is applied where different pairs from $N$ number of parts (each with $k$ number of tooling elements) are formed and superimposed to each other through translation and rotation in a Cartesian coordinate system to minimise the residual error or accumulative difference between two fixture configurations in the pair. In order to speed up the optimization, pairing is done such that a pair comprises of a part and the centroid of the remaining $N-1$ parts.

Once the optimal superimposition of different configurations is obtained, the diameter of fixture workspace envelope (FWE) is determined by minimizing the diameter of the largest circle (out of $k$ circles) containing $N$ tooling elements (locating points) corresponding to each part. The diameter of the largest circle is minimized through making $N$ pairs, each with one locating point and centroid of the rest of the $N-1$ locating points and conducting search optimization for this configuration pair to minimize the diameter of the largest circle (say $i$ th one).

The objective function for the fixture configuration optimization is given by

$$
\operatorname{Min}\left(\operatorname{Max} C_{i}\right) \quad 1 \leq i \leq k .
$$


Though this deterministic Procrustes-based pairwise optimization achieves the optimization of fixture design, the absence of proper ordering of pair formation in each iteration impacts the computational time severely. An unplanned ordering strategy for each search optimization to compress the two members of the pair would result in the exact optimal solution at the cost of computational time. In order to impart further momentum to early supplier involvement, the computational time should be given priority over exact optimality of the solution. For this purpose, this paper randomizes the search procedure as follows.

(a) During each iteration of Procrustes analysis, the pair formation is generated by randomly selecting a locating point and taking the centroid of $N-1$ locating points. It is ensured, in each of the iterations, that the point which is once selected should not be selected again. Once $N$ pair formations is followed by sum of squares of each pair of configuration, one iteration is completed. Repeating Procrustes analysis in this way yields a near optimal/optimal superimposition of part configuration.

The sum of squares of each pair of configuration [2] is given by

$$
\begin{aligned}
D & \left(X_{1}, X_{2}, \ldots, X_{n},\right) \\
& =\frac{1}{N} \sum_{i=1}^{N} \sum_{j=i+1}^{N}\left\|\left(X_{i} R_{i}+l_{k} T_{i}\right)-\left(X_{j} R_{j}+l_{k} T_{j}\right)\right\|^{2},
\end{aligned}
$$

where $X_{i}$ is the configuration matrix, $R_{i}$ and $T_{i}$ are the rotation and translation vectors, and $l_{k}$ is the matrix with all the tooling elements of a part configuration.

(b) After determination of the superimposition of the part configuration, the diameter of the largest circle containing $N$ parts is minimized. This is accomplished by pairwise optimization [2] as told above. Computational economy with respect to time can be brought about to this procedure by stochastically selecting a part while doing any pair formation in each of the iterations. This can be done by calculating the distance of locating elements from the centre of the circle and normalizing them. The normalized distance of a locating point is used in deciding selection of a point for the pair formation with the rest of $N-1$ points. That is, a point with lesser value normalized distance is selected first for pair formation and that with higher value is selected later.

This extends the Procrustes-based pairwise optimization by imparting to it a stochastic outlook. This helps in obtaining a sub optimal solution in a stipulated time frame.

The extended Procrustes-based pairwise optimization is implemented over an example taken from Kong and Ceglarek [2], and the stopping criterion for the algorithm is kept as the maximum number of generation. Different trials are preformed, and the diameter obtained versus trial number plot is drawn as is shown in Figure 7.

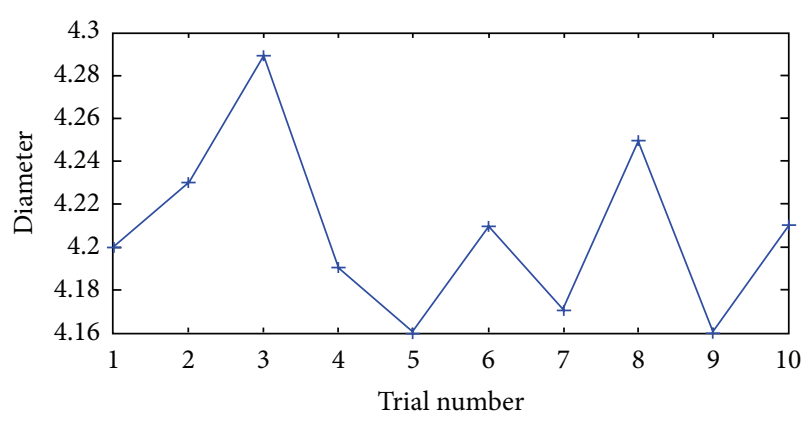

Figure 7: Performance of stochastic Procrustes-based pairwise optimization for stipulated number of iterations or time frame.

As reflected from the figure, for most of the trials the diameter of the largest circle is between what is found by Lee et al's approach or Kong and Ceglarek approach. Moreover, only once the diameter value found by stochastic Procrustesbased pairwise optimization is equal to 4.29 otherwise it is near to 4.16 units. Thus, the stochastic Procrustes-based pairwise optimization algorithm results in a viable solution in stipulated time.

\section{Conclusions and Future Research}

This paper attempts to conceptualize the product development of a product family in a reconfigurable assembly system in which product development is performed concurrently with fixture design. The fixture design is accomplished as per the parts' requirements by internal or external suppliers, and active interaction is simulated through a web-based interface so as to have early supplier involvement. The proposed webbased interface involves four steps of interaction where two of them are concerned with optimization issues. The first optimization problem is related to fixture designer selection by the product designers, while the latter one is optimization of fixture design by the fixture designer. To simulate promptness to the interaction between the two members, both of the optimizations can be aided with stochastic optimization algorithm.

Though the cooperation and integration of the product designer and fixture designer activities promise to maximize the market share, this aspect is not analyzed explicitly by this paper, thus being a future scope of research. Moreover, agents can be utilized more compactly to imbue sophistications and intelligence in the communication.

As synchronous knowledge share, an agent communication platform based on Java Agent Template Lite is built to enable online communication between the product designer and the fixture designer, and being used by a user group without any complain so far, the efficacy of the proposed digital interface can be established. At the same time the multiple-supplier selection problem is also demonstrated to be near optimally solved using a sophisticated algorithm in realistic computational time. Holistically, we are encompassing both of the products dependent and nonproduct dependent part in general. A more discreet and decisive 
model for reconfigurable or nonreconfigurable models is an area of further research.

\section{References}

[1] M. V. Martin and K. Ishii, "Design for variety: developing standardized and modularized product platform architectures," Research in Engineering Design, vol. 13, no. 3, pp. 213-235, 2002.

[2] Z. Kong and D. Ceglarek, "Fixture workspace synthesis for reconfigurable assembly using procrustes-based pairwise configuration optimization," Journal of Manufacturing Systems, vol. 25, no. 1, pp. 25-38, 2006.

[3] A. M. Choubey, P. Prakash, F. T. S. Chan, and M. K. Tiwari, "Solving a fixture configuration design problem using genetic algorithm with learning automata approach," International Journal of Production Research, vol. 43, no. 22, pp. 4721-4743, 2005.

[4] K. Bastani, Z. Kong, W. Huang, X. Huo, and Y. Zhou, "Fault diagnosis using an enhanced Relevance Vector Machine (RVM) for partially diagnosable multistation assembly processes," IEEE Transactions on Automation Science and Engineering, vol. 10, no. 1, pp. 124-136, 2013.

[5] J. Camelio, S. J. Hu, and D. Ceglarek, "Modeling variation propagation of multi-station assembly systems with compliant parts," Journal of Mechanical Design, Transactions of the ASME, vol. 125, no. 4, pp. 673-681, 2003.

[6] Z. Kong, D. Ceglarek, and W. Huang, "Multiple fault diagnosis method in multistation assembly processes using orthogonal diagonalization analysis," Journal of Manufacturing Science and Engineering, Transactions of the ASME, vol. 130, pp. 1-10, 2008.

[7] Y. Liu, S. Jin, Z. Lin, C. Zheng, and K. Yu, "Optimal sensor placement for fixture fault diagnosis using Bayesian network," Assembly Automation, vol. 31, no. 2, pp. 176-181, 2011.

[8] Z. Kong and D. Ceglarek, "Rapid deployment of reconfigurable assembly fixtures using workspace synthesis and visibility analysis," CIRP Annals, vol. 52, no. 1, pp. 13-16, 2003.

[9] A. Millar and H. Kihlman, "Reconfigurable flexible tooling for aerospace wing assembly," in Proceedings of the SAE AeroTech Congress \& Exhibition, Seattle, Wash, USA, November 2009.

[10] D. Tang, W. Eversheim, G. Schuh, and K.-S. Chin, "CyberStamping: a web-based environment for cooperative and integrated stamping product development," International Journal of Computer Integrated Manufacturing, vol. 17, no. 6, pp. 504-519, 2004.

[11] D. Tang, "An agent-based collaborative design system to facilitate active die-maker involvement in stamping part design," Computers in Industry, vol. 54, no. 3, pp. 253-271, 2004.

[12] W. Eversheim, M. Westekemper, and F. Spennemann, "Cooperation with tool and fixture supplier," in Strategic Production Networks, L. Zheng and F. Posseloleken, Eds., pp. 176-187, Springer, Berlin, Germany, 2002.

[13] P. C. Stadzisz and J.-M. Henrioud, "An integrated approach for the design of multi-product assembly systems," Computers in Industry, vol. 36, no. 1-2, pp. 21-29, 1998.

[14] E. Travaini, P. Pedrazzoli, R. Rinaldi, and C. R. Boër, "Methodological approach and reconfiguration tool for assembly systems," CIRP Annals, vol. 51, no. 1, pp. 9-13, 2002.

[15] Y. Rong and Y. Bai, "Machining accuracy analysis for computeraided fixture design verification," Journal of Manufacturing Science and Engineering, Transactions of the ASME, vol. 118, no. 3, pp. 289-300, 1996.
[16] N. Kaya, "Machining fixture locating and clamping position optimization using genetic algorithms," Computers in Industry, vol. 57, no. 2, pp. 112-120, 2006.

[17] D. Tang and B. Gao, "Feature-based metal stamping part and process design-part I: stampability evaluation," International Journal of Production Research, vol. 45, no. 12, pp. 2673-2695, 2007.

[18] G. C. Vosniakos and T. Giannakakis, "A knowledge-based manufacturing advisor for pressworked sheet metal parts," Journal of Intelligence Manufacturing, 2012.

[19] S. Kumar, R. Singh, and G. S. Sekhon, "CCKBS: a component check knowledge-based system for assessing manufacturability of sheet metal parts," Journal of Materials Processing Technology, vol. 172, no. 1, pp. 64-69, 2006.

[20] W. Hu, L. G. Yao, and Z. Z. Hua, "Optimization of sheet metal forming processes by adaptive response surface based on intelligent sampling method," Journal of Materials Processing Technology, vol. 197, no. 1-3, pp. 77-88, 2008.

[21] M. Firat, "Computer aided analysis and design of sheet metal forming processes-part 3: stamping die-face design," Materials and Design, vol. 28, no. 4, pp. 1311-1320, 2007.

[22] J.-M. Huang, Y.-T. Jou, L.-C. Zhang, S.-T. Wang, and C.-X. Huang, "A web-based model for developing: a mold base design system," Expert Systems with Applications, vol. 36, no. 4, pp. 8356-8367, 2009.

[23] J. Li and D. Su, "Support modules and system structure of webenabled collaborative environment for design and manufacture," International Journal of Production Research, vol. 46, no. 9, pp. 2397-2412, 2008.

[24] Y. Yujun, Z. Jinsong, W. Li, and C. Liping, "Internet-based collaborative product development chain for networked product development," International Journal of Advanced Manufacturing Technology, vol. 28, no. 7-8, pp. 845-853, 2006.

[25] G. Q. Huang, J. B. Zhao, and K. L. Mak, "Early supplier involvement in new product development with WeBid: a case study on video conferencing system," Journal of Computing and Information Science in Engineering, vol. 3, no. 4, pp. 363-365, 2003.

[26] G. Q. Huang and K. L. Mak, "Brokering the customer-supplier partnership in product design and realization over the World Wide Web," IIE Transactions, vol. 35, no. 4, pp. 369-378, 2003.

[27] P. Gupta and R. Nagi, Optimal partner selection for virtual enterprises in agile manufacturing [thesis], Department of Industrial Engineering, SUNY at Buffalo, Buffalo, NY, USA, 1995.

[28] D. Y. Sha and Z. H. Che, "Supply chain network design: partner selection and production/distribution planning using a systematic model," Journal of the Operational Research Society, vol. 57, no. 1, pp. 52-62, 2006.

[29] Z. Lin and L. Wang, "Agile partner selection based on metaheuristic algorithms," in Proceedings of the IEEE International Conference on e-Business Engineering (ICEBE '08), pp. 402-407, October 2008.

[30] H. Jeon, C. Petrie, and M. R. Cutkosky, "JATLite: a Java agent infrastructure with message routing," IEEE Internet Computing, vol. 4, no. 2, pp. 87-96, 2000.

[31] L. N. de Castro and J. I. Timmis, Artificial Immune Systems: A New Computational Intelligence Approach, Springer, London, UK, 2002.

[32] L. N. De Castro and F. J. Von Zuben, "Learning and optimization using the clonal selection principle," IEEE Transactions on Evolutionary Computation, vol. 6, no. 3, pp. 239-251, 2002. 
[33] C.-Y. Tsai, C.-C. Lo, and A. C. Chang, "Using fuzzy QFD to enhance manufacturing strategic planning," Journal of the Chinese Institute of Industrial Engineers, vol. 20, no. 1, pp. 3341, 2003.

[34] J. Lee, S. J. Hu, and A. C. Ward, "Workspace synthesis for flexible fixturing of stampings," Journal of Manufacturing Science and Engineering, Transactions of the ASME, vol. 121, no. 3, pp. 478$484,1999$. 


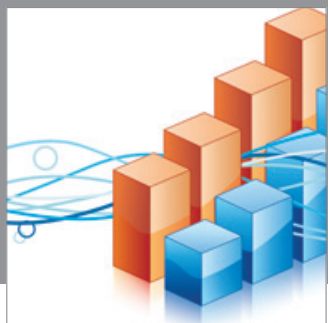

Advances in

Operations Research

mansans

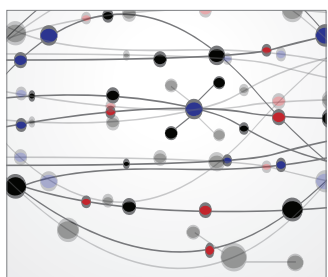

The Scientific World Journal
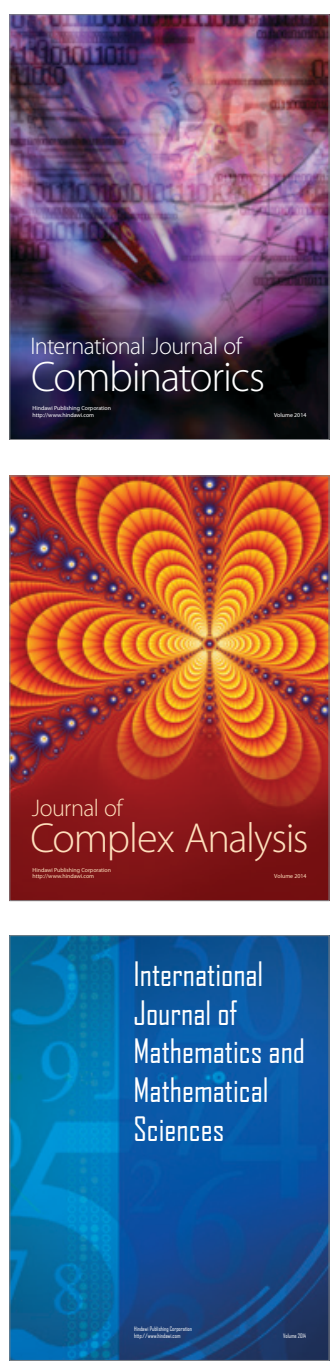
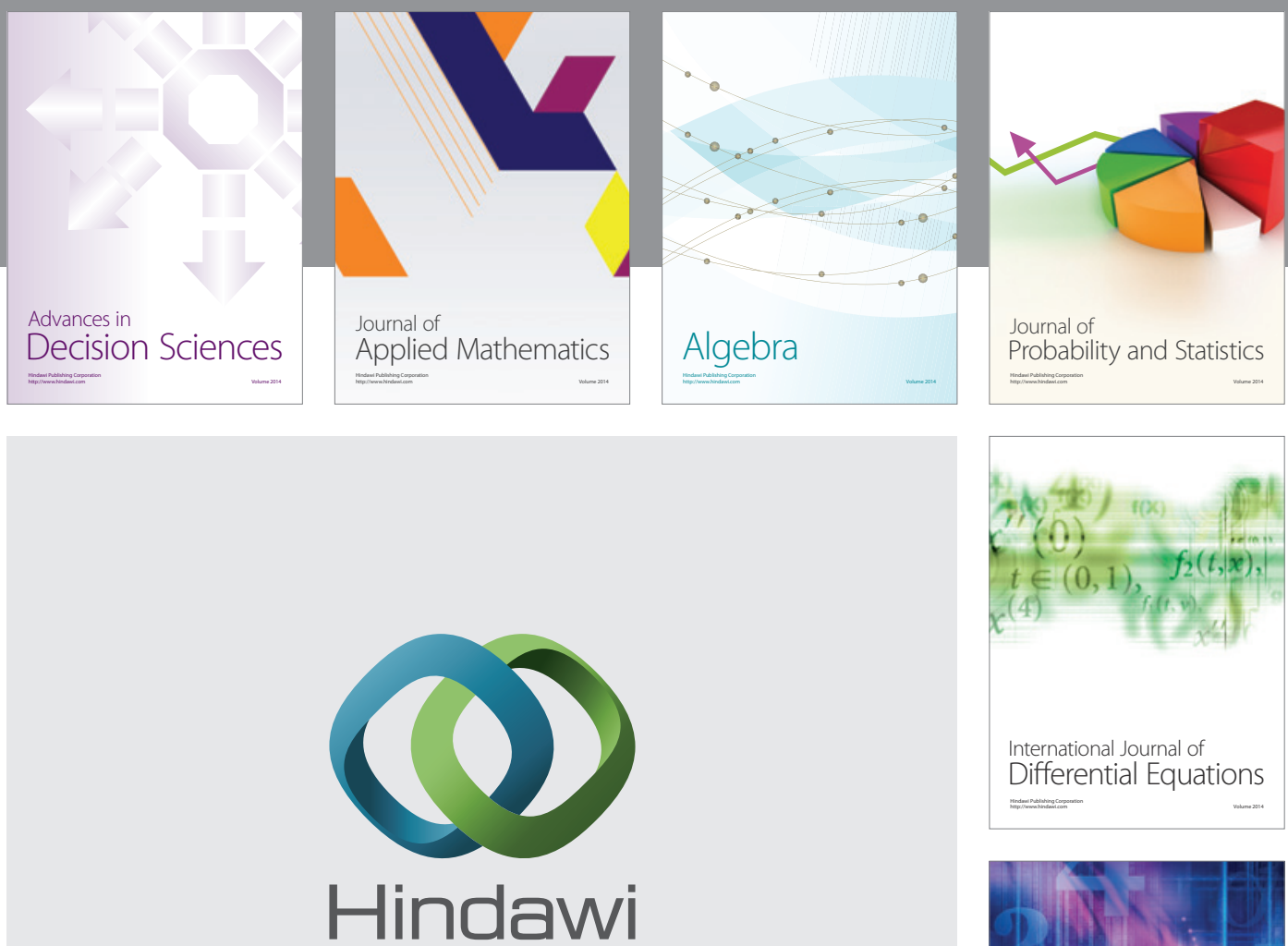

Submit your manuscripts at http://www.hindawi.com
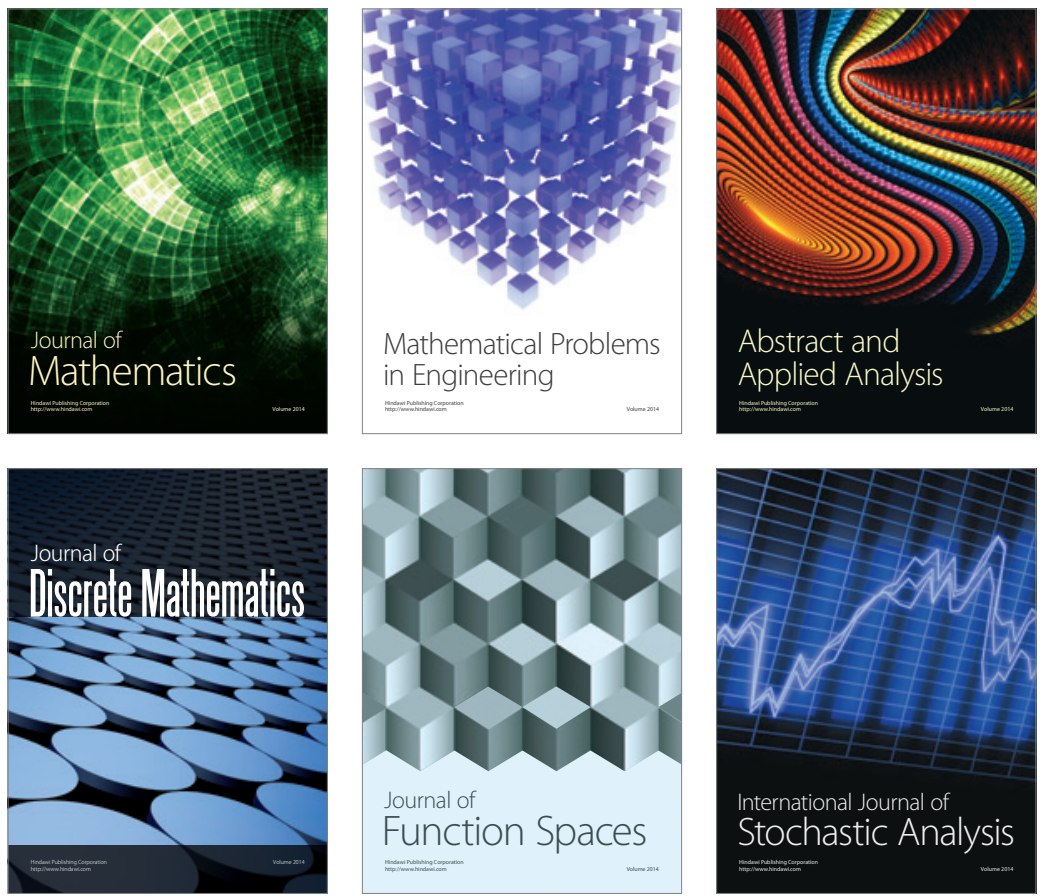

Journal of

Function Spaces

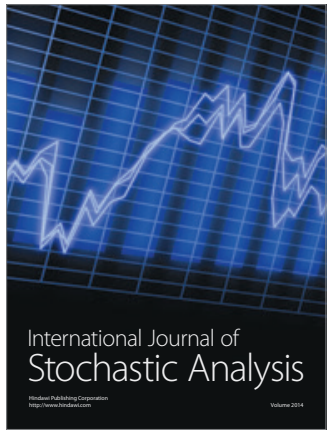

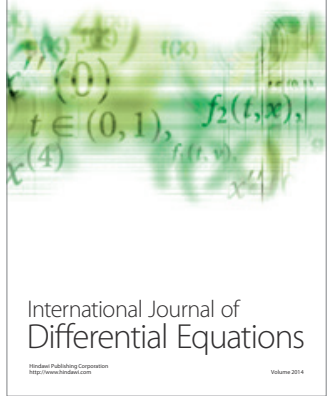
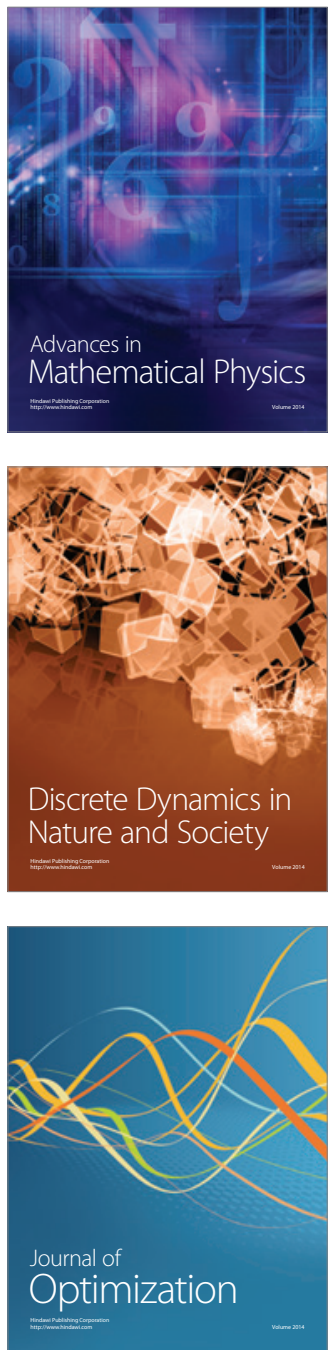